\title{
ATLAST-9.2m: a large-aperture deployable space telescope
}

\author{
William R. Oegerle ${ }^{a}$, Lee D. Feinberg ${ }^{a}$, Lloyd R. Purves ${ }^{a}$, T. Tupper Hyde ${ }^{a}$, Harley A. Thronson ${ }^{a}$,
} Jacqueline A. Townsend ${ }^{a}$, Marc Postman ${ }^{b}$, Matthew R. Bolcar ${ }^{a}$, Jason G. Budinoff ${ }^{\mathrm{a}}$, Bruce H. Dean ${ }^{\mathrm{a}}$, Mark C. Clampin ${ }^{a}$, Dennis C. Ebbets ${ }^{c}$, Qian Gong ${ }^{a}$, Theodore R. Gull ${ }^{\mathrm{a}}$, Joseph M. Howard ${ }^{\mathrm{a}}$, Andrew L. Jones ${ }^{a}$, Richard G. Lyon ${ }^{a}$, Bert A. Pasquale ${ }^{a}$, Charles Perrygo ${ }^{a}$, Jeffrey S. Smith ${ }^{a}$, Patrick L. Thompson $^{\mathrm{a}}$, Bruce E. Woodgate ${ }^{\mathrm{a}}$

${ }^{a}$ NASA Goddard Space Flight Center, Greenbelt, MD USA 20771;

${ }^{\mathrm{b}}$ Space Telescope Science Institute, 3700 San Martin Drive, Baltimore, MD USA 21218;

${ }^{\mathrm{c}}$ Ball Aerospace \& Technologies Corp, 1600 Commerce St, Boulder, CO USA 80301

\begin{abstract}
We present results of a study of a deployable version of the Advanced Technology Large-Aperture Space Telescope (ATLAST), designed to operate in a Sun-Earth L2 orbit. The primary mirror of the segmented 9.2-meter aperture has 36 hexagonal $1.315 \mathrm{~m}$ (flat-to-flat) glass mirrors. The architecture and folding of the telescope is similar to JWST, allowing it to fit into the $6.5 \mathrm{~m}$ fairing of a modest upgrade to the Delta-IV Heavy version of the Evolved Expendable Launch Vehicle (EELV). We discuss the overall observatory design, optical design, instruments, stray light, wavefront sensing and control, pointing and thermal control, and in-space servicing options.
\end{abstract}

Keywords: Large space telescopes, deployable telescopes, telescope servicing

\section{INTRODUCTION}

The Advanced Large Aperture Space Telescope (ATLAST) is a concept for a next generation flagship astrophysics mission to study the universe at ultraviolet (UV), optical and near-infrared (NIR) wavelengths - essentially the same portion of the spectrum observed by the Hubble Space Telescope (HST). However, ATLAST, with a primary mirror diameter of $8-16 \mathrm{~m}$ and diffraction limited at $\lambda=500 \mathrm{~nm}$, would have a 1-D spatial resolution 4-10 times better than HST, and significantly larger collecting area. This would enable a wide variety of science at the forefront of astrophysics, including characterization of the atmospheres of earth-mass exoplanets, precise determination of the stellar initial mass function, observations of the mass assembly of galaxies, characterization of the intergalactic medium and physics at the center of active galaxies. See further details, see papers on science with ATLAST in this conference, the 2008 SPIE conference ${ }^{1}$, as well as the public ATLAST website: http://www.stsci.edu/institute/atlast.

Several different concepts for ATLAST have been developed: (1) an $8 \mathrm{~m}$ monolithic primary mirror concept ${ }^{2}$ and (2) a $16 \mathrm{~m}$ segmented mirror design. Both concepts require a 9-10 $\mathrm{m}$ diameter fairing and a heavy lift launch vehicle. Given the uncertainty surrounding the development timescale for such launch vehicles, it was decided to also develop a concept for ATLAST that could be launched with an Evolved Expendable Launch Vehicle (EELV). In this paper, we describe the design of the resulting observatory, called the ATLAST-9.2, which has a 9.2m diameter segmented-primary mirror.

\section{ATLAST-9.2 DESIGN}

ATLAST-9.2 retains significant heritage from the James Webb Space Telescope (JWST), thereby taking advantage of technologies and engineering already developed for that mission. Hence, we adopted a segmented-mirror architecture and a folding similar to JWST. However, the telescope in not cryogenic and is planned to operate at approximately room temperature, thereby greatly simplifying observatory testing on the ground. This also simplifies the design of the sunshield, whose purpose for ATLAST-9.2 is solely to block the light from the sun, moon and earth at Sun-Earth Lagrange point 2 (SEL2), and not to act as a thermal shield for a cryogenic telescope. The primary challenge for ATLAST-9.2 is in the demands placed on optics and wavefront control due to the requirement of diffraction-limited imaging at a wavelength of $500 \mathrm{~nm}$. 


\subsection{Launch Vehicle}

The allowable size of the primary mirror was determined from a consideration of an achievable EELV fairing size. For this study we assumed that a fairing with outer/inner diameter of $6.5 / 6.0 \mathrm{~m}$ could become available in the near future. Indeed, the United Launch Alliance has indicated that such a fairing size had been evaluated and appeared to be feasible for the Delta IV Heavy vehicle ${ }^{3}$. ATLAST-9.2 is not dependent on the Delta IV - the ATLAS V or other such vehicles are also suitable if their fairing sizes and lift capacities are sufficient. Nevertheless, we have assumed the Delta-IV Heavy vehicle in our concept study outfitted with six solid booster rockets and the RS68A version of the main engine. The solid boosters are already in use on smaller Delta IVs, and the upgraded main engine is already in testing, with its first flight expected in the near future. A graphic showing the folded ATLAST-9.2 observatory in the fairing is shown in Figure 1.

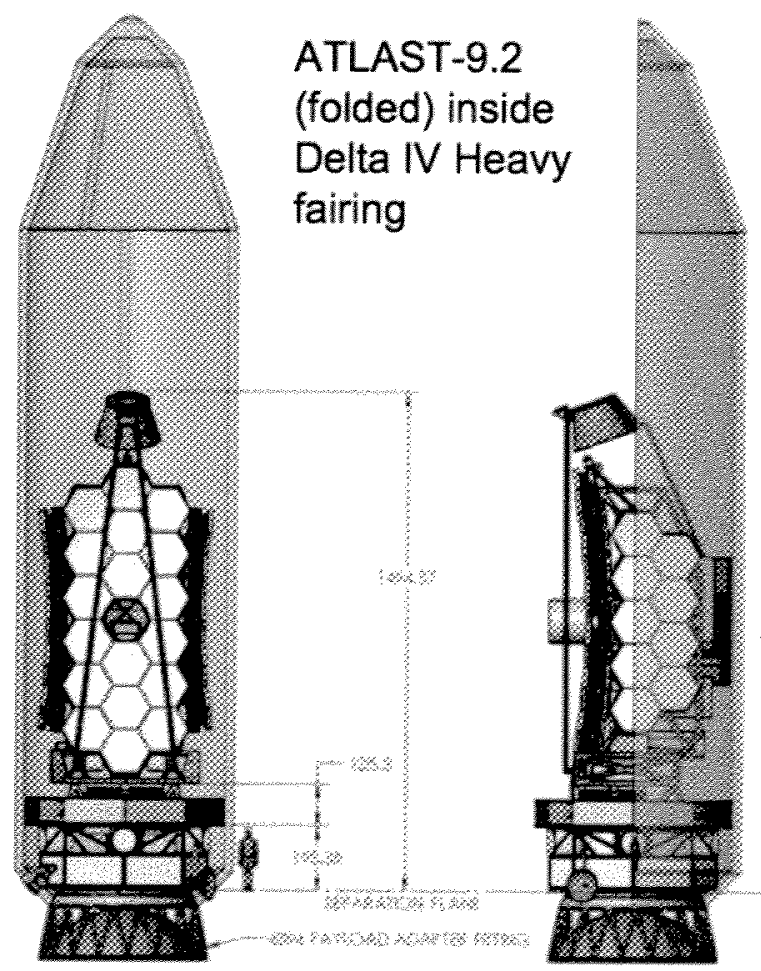

Figure 1. A picture of the folded and stowed ATLAST-9.2 observatory in the $6.5 \mathrm{~m}$ outer-diameter fairing of the Delta IV Heavy launch vehicle. The fairing is $29.5 \mathrm{~m}$ in height.

\subsection{Optical Telescope Assembly and Optical Design}

The primary mirror consists of 36 segments that are $1.315 \mathrm{~m}$ in size, flat-to-flat. The segments are the same size as those of JWST, but we have baselined the use of ULE glass instead of Beryllium. Beryllium mirrors were chosen for JWST for their superior coefficients of thermal expansion (CTE) at cold temperatures, but that is not a consideration for ATLAST which operates at room temperature. Furthermore, at the present time, ULE shows the most promise for excellent quality wavefront error (WFE). Dahl $1^{4}$ has reported $15 \mathrm{~nm}$ WFE for a $1.4 \mathrm{~m}$ ULE mirror segment under the MMSD program. We also baselined smaller segments, instead of fewer large segments, in order to maintain the stiffness of each segment. The approach uses JWST's 7-degree-of-freedom architecture (a hexapod plus radius of curvature actuator), which minimizes the overall cost and complexity of the actuation electronics and control. Lightweight mirror segments are of course advantageous, and the MMSD mirrors have areal densities of $\sim 10 \mathrm{~kg} / \mathrm{m}^{2}$, compared to the earlier ULE AMSD $/ J W S T$ mirrors that had achieved $15 \mathrm{~kg} / \mathrm{m}^{2}$. However, during our study, we conservatively baselined segments with areal density of $25 \mathrm{~kg} / \mathrm{m}^{2}$ for several reasons: (1) we wanted to ensure mirror stiffness for wavefront 
quality and (2) we were somewhat concerned about the potential damage to ultra-lightweight mirrors from micro-meteor impacts. As part of the ATLAST technology development program, we hope to achieve very stiff, lightweight mirror segments with excellent figure. The $9.2 \mathrm{~m}$ aperture has collecting area of $50 \mathrm{~m}^{2}$, so reduction of areal density by 15 $\mathrm{kg} / \mathrm{m}^{2}$ not only saves $750 \mathrm{~kg}$ in mirror mass, but also significant mass in the backplane and support structure. The stowed and deployed optical telescope assembly (OTA) is shown in Figure 2.

The ULE mirror architecture proposed for ATLAST-9.2 has a large staffing and facility infrastructure in place at ITT, Tinsley and Corning, so very little facility modifications are required. This is important because modifications are expensive and consume schedule. Segmented mirror architecture does offer the unique advantage of allowing parallel polishing of the mirrors. This, in turn, allows more time to be spent polishing each segment, and enables the very smooth mirror surfaces required for both UV and high contrast applications.

The secondary mirror for ATLAST-9.2 is made out of ULE and has comparable requirements to the primary mirror, the key difference being it can be stiffer because it is smaller and can have higher mass since it is just one mirror. Final performance is more challenging because it is convex and, thus, cannot be tested at center of curvature. Its performance will be completely determined from metrology. The secondary mirror assembly employs a six-degree-of-freedom hexapod, which is a smaller version of that used on the primary mirror.
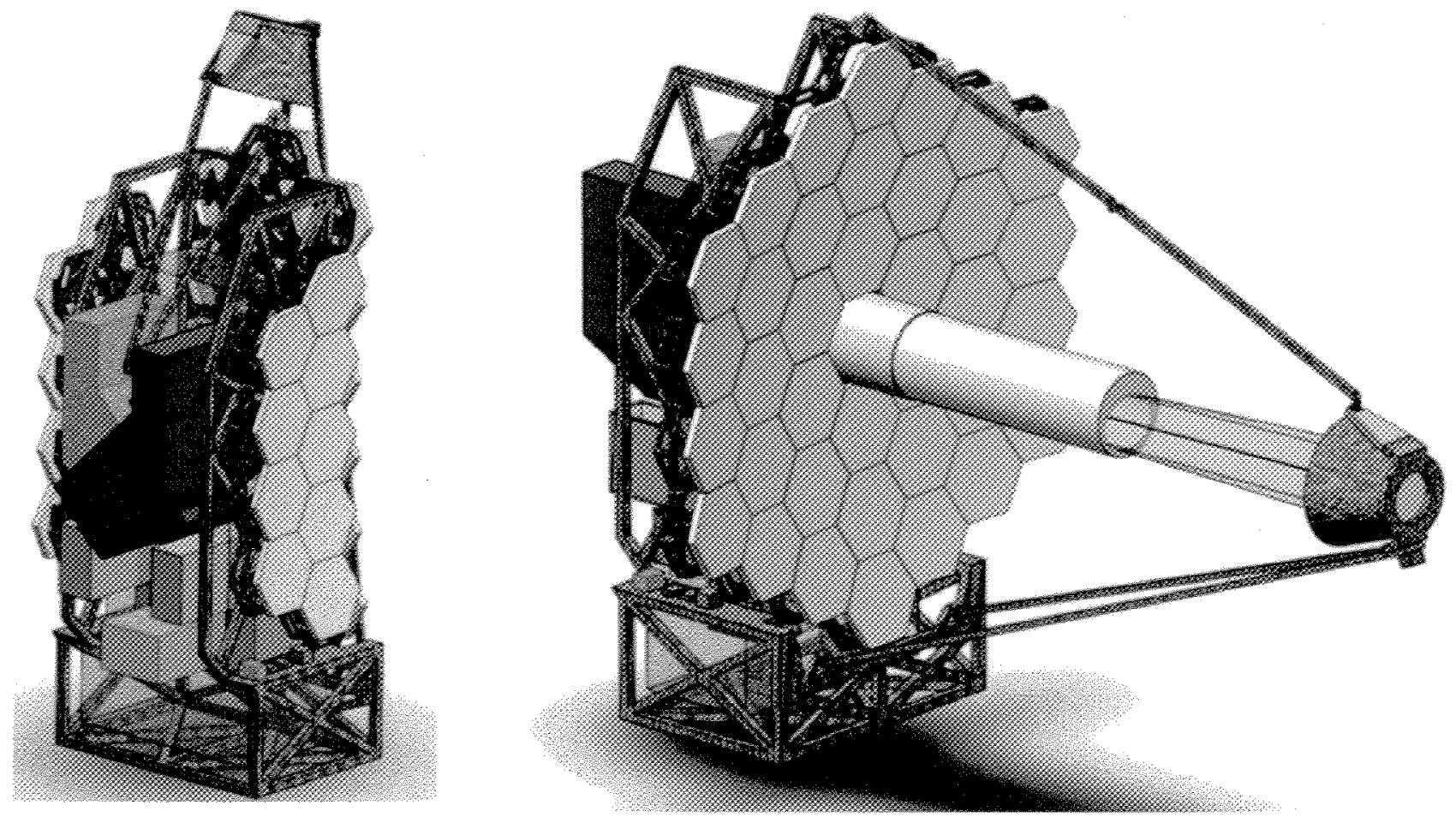

Figure 2. The folded and stowed OTA (left) showing the location of instruments (colored boxes), and the deployed OTA (right). Note the central primary light baffle for the control of stray light.

The optical design is a modified Three-Mirror Anastigmat (TMA) that provides a channel for wide FOV instruments and a Cassegrain channel that minimizes reflections in the UV. The primary mirror is fast ( $f / 1.25)$ to minimize the length of the OTA. The primary and secondary mirrors are coated with $\mathrm{Al}+\mathrm{MgF}_{2}$ for $\mathrm{UV}$ response. The Cassegrain channel contains coronagraphic instruments for observing exoplanets, as well as UV spectrographs. In the current design, the WFE in the Cass channel is within the required range over only a relatively small field of view - about 1 arcmin in diameter. However, this is large enough for most UV spectroscopic work and certainly large enough for exoplanetary systems. All optics in the TMA channel and in the exoplanet instruments with $\lambda>500 \mathrm{~nm}$ are coated with protected 
silver. The design is diffraction-limited at $\lambda=500 \mathrm{~nm}$ over an $8 \times 20$ arcmin FOV. The wavefront error map for the TMA channel is shown in Figure 3.

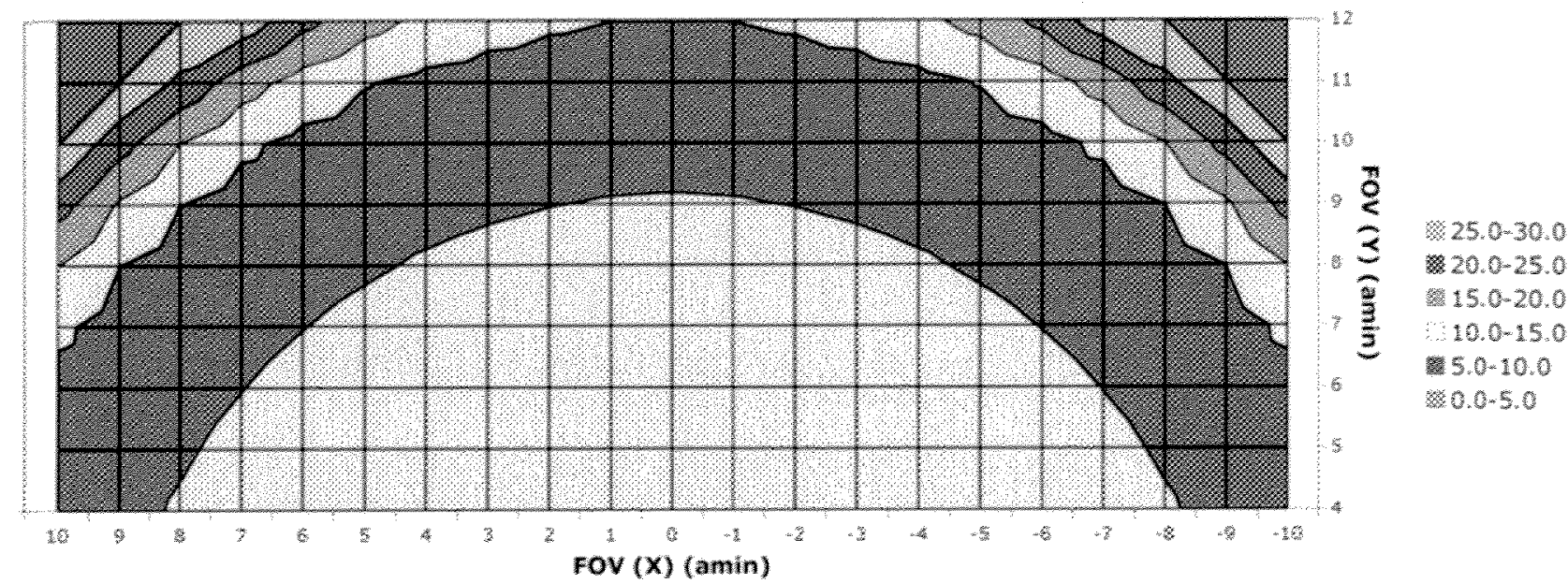

Figure 3. The wavefront error map in the TMA channel showing that wavefront errors are confined to $5-10 \mathrm{~nm}$ $\mathrm{rms}$ over a relatively large field of view of $\sim 8 \times 20$ arcmin. Legend on right shows units in $\mathrm{nm}$ (color-coded).

The backplane structure for ATLAST-9.2 is a cyanate ester graphite epoxy. Although there is an active control system for the OTA, the stability requirement is comparable to JWST to minimize required updates. We have baselined the same material used on high-performance UV instrument benches, such as those used on HST, for the composite laminate. However, some of the techniques used to achieve better thermal stability on JWST (improved modeling and coupon testing, bond-line control, etc.) will be employed on ATLAST-9.2 along with active thermal control. The structure for the OTA also houses the instruments as was done on HST, providing a single optical truss structure. The entire structure makes use of heaters as needed for active thermal control as was done on HST. To reduce electrical power requirements, heat pipes conduct waste heat from the instruments and electronics to the primary mirror. Heater panels behind the primary mirror segments actively control the segment temperature to $\pm 0.1 \mathrm{deg} \mathrm{K}$, and any morphology changes due to spatial/thermal gradients are addressed by the wavefront sensing and control system.

Fine guidance and wavefront sensing on ATLAST-9.2 will be performed by four nearly identical instruments called "hybrid" instruments that draw heavily on algorithms verified for the JWST. Light from the OTA is fed to the hybrid instrument via a pick-off mirror. A bi-directional star-selection mirror is used to access a $4 \times 4$ arcmin field-of-view to steer an isolated, bright star onto the FGS and WFS detectors. The beam of light is split three ways: $20 \%$ of the light goes to the FGS detector (for guidance) and $40 \%$ goes to each of two WFS detectors for wavefront sensing. The FGS beam path consists only of imaging optics to form a broadband image of the star on the detector for centroiding and guidance. Each WFS beam path contains a dual filter wheel and imaging optics to form a narrowband, diversity defocus image of the star on each of the WFS detectors. One of the two WFS beam paths also contains an actuated pupilimaging lens that may be inserted to create an image of the OTA pupil on the detector. A star as faint as V 19 magnitude provides enough photons to allow computation of wavefront error every $\sim 10$ minutes. The WFS algorithm is an iterative hybrid-diversity phase-retrieval algorithm similar to what has been previously developed for the JWST ${ }^{5}$. Commands are then sent autonomously to make needed adjustments to the mirror segments. The actuators on all 36 segments can be moved simultaneously, providing a quick adjustment. The whole process runs continuously while under FGS control to minimize wavefront error in a near-real time. Feinberg et al ${ }^{6}$ has provided a detailed description of the wavefront sensing process and the hybrid instrument.

\subsection{Instruments}

The principal imaging camera in ATLAST-9.2 is a wide field-of-view panchromatic camera in the TMA channel. This camera covers the wavelength range from $450 \mathrm{~nm}$ to at least $1700 \mathrm{~nm}$ (and potentially out to $2500 \mathrm{~nm}$ with cooling) with a field of view (FOV) of $8 \times 8$ arcmin. A dichroic splits the incoming beam into a visible CCD channel covering the 450 - 
$900 \mathrm{~nm}$ range, and a near-IR HgCdTe channel covering 900-1700/2400 nm. The visible channel covers the FOV with a $4 \times 4$ array of $8 \mathrm{~K} \times 8 \mathrm{~K}$ CCDs $(1 \mathrm{Gpixel})$ with detector pixel size of 9 microns and critical sampling of images $(2$ pixels per Airy spot at $\lambda=500 \mathrm{~nm})$. The NIR channel covers the same FOV with a $4 \times 4$ array of $4 \mathrm{~K} \times 4 \mathrm{~K} \mathrm{HgCdTe}$ arrays $(0.25 \mathrm{Gpix})$ with detector pixel size of 18 microns and critical sampling at $\lambda=1.1$ microns. Refractive components have been selected for the collimator and imagers to dramatically reduce the total volume and component sizes, as well as to improve image quality in a wide FOV. The optical layout for the camera is shown in Figure 4. Assuming that for typical observations of deep fields, the CCDs would be read-out every 1000 seconds and the NIR detectors every 100 seconds, for a continuous data rate of $4.1 \mathrm{MB} / \mathrm{sec}$ (compressed 2:1), or $0.35 \mathrm{~TB}$ per day.

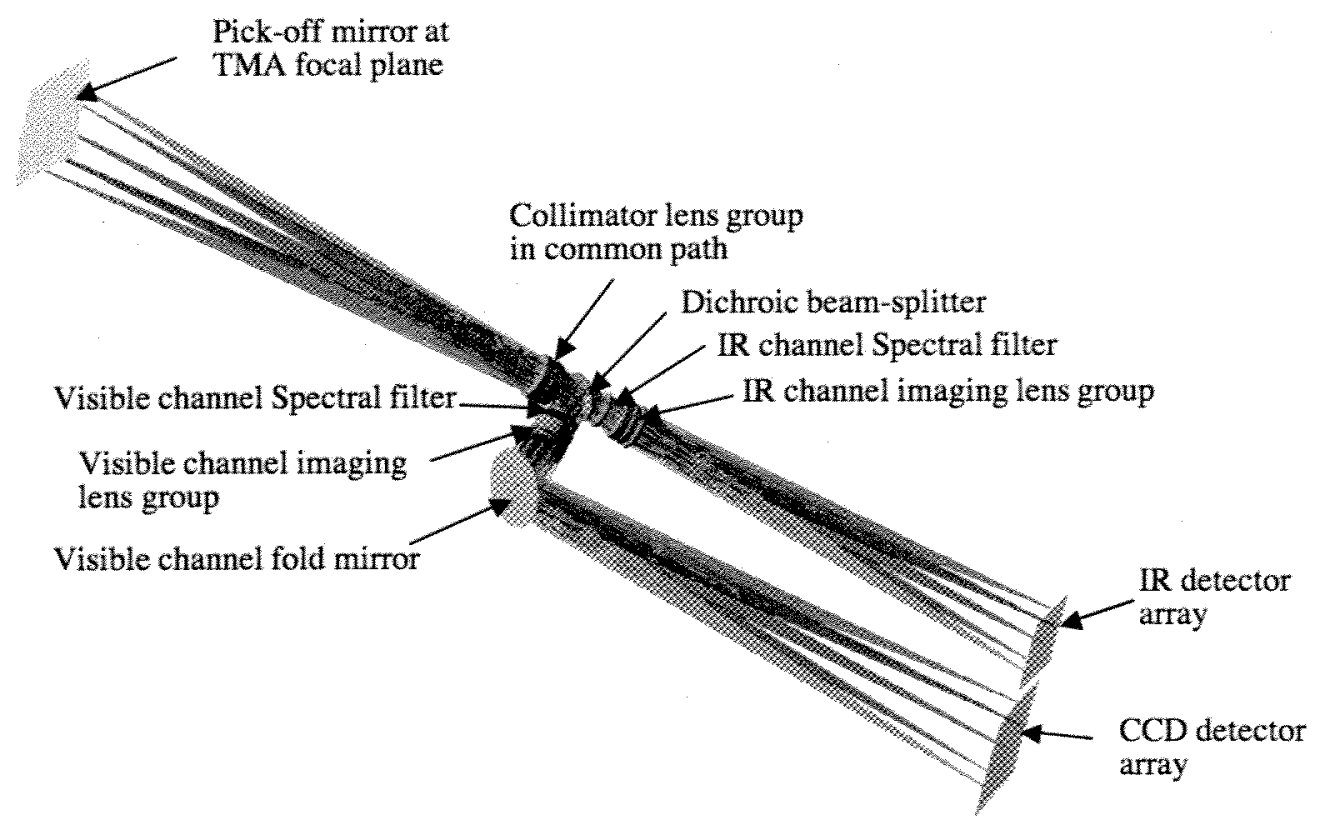

Figure 4. Optical layout of the wide field-of-view camera in the TMA channel. Light is split into a visible and NIR channel by a beam splitter and re-imaged for proper image sampling.

We have also baselined a Visible/NIR multi-object spectrograph (MOS) in the TMA channel, for relatively wide-field spectroscopy of galaxies, stars and AGN to study the intergalactic medium, star formation, and galaxy evolution. As in the imaging camera, light is split into visible and NIR channels with a beam splitter, and then passes through a mosaic of programmable microshutter arrays (similar to the ones employed in NIRSpec on JWST) for selection of many hundreds of objects to be dispersed onto the detectors, with resolving power set by selectable gratings and prisms. The use of photon counting detectors could maximize the science at higher spectral resolution where signal-to-noise is set by detector performance and not the sky noise. Such detectors are becoming available in the visible, and are under development in the NIR. The MOS could be used in parallel mode while observing a different field in the wide FOV camera.

In the Cassegrain channel, the primary science instruments include an exoplanet instrument and a UV integral field spectrograph. The exoplanet instrument has essentially two modes: an imager (ExoCam) and a spectrograph (ExoSpec). For exoplanet observations, the instrument operates behind a starlight suppression system - either an internal coronagraph or an external occulter. When used with the external occulter, the optical beam is taken directly from the telescope optics. This optical path can also be used without the occulter for general astrophysics observations. For use with an occulter, the first mirror, at the telescope focal plane, is a dichroic, allowing longer wavelengths to feed a shadow sensor instrument that helps to determine the telescope's lateral location within the occulter shadow. Since ATLAST-9.2 has a segmented primary mirror, we have adopted the visible nulling coronagraph (VNC) to provide an internal capability for starlight suppression. If exoplanets are detected, then the ExoSpec mode can be used to obtain spectroscopic data of them. The Cassegrain channel can be split into multiple channels through the use of beam splitters 
in order to maximize the science at different wavelengths. For instance, a near UV channel will permit the search for ozone and Rayleigh scattering in exoplanet atmospheres.

We have also considered a UV integral field spectrograph (UV IFS) for the Cassegrain channel, with resolving power of 100 to 100,000 . An all-reflective concept covers a $1 \times 1$ arc sec field with 27 milli-arsec spatial samples. A mapping capability would be possible using lens-lets or mirror-lets providing $30 \times 30$ pixel mappings. In order to maximize UV throughput, the number of optical surfaces or elements in the UV IFS is minimized. A relay mirror and an all-reflective image slicer provide the 2D capability. A method combining 2D spatial information with high throughput uses a reflective image slicer at the telescope focus, with each slice serving a concave grating, and all gratings feeding a common detector (credit: Ball Aerospace design).

Certainly, other instruments would be proposed for inclusion on ATLAST-9.2, per NASA's custom of competing instruments on flagship missions. Such solicited instruments could improve or entirely replace those baselined here. Our study was aimed at providing a scientifically compelling and feasible design, but not necessarily the optimal design.

\section{ATLAST-9.2 MISSION DESIGN}

\subsection{Deployment}

The folded version of ATLAST-9.2 is shown in Figure 1. After fairing separation, the observatory is deployed as shown in Figure 5. The solar array is deployed first, followed by the high gain antenna, the sunshield and then the gimballed pointing arm and OTA.

\section{Deployment Sequence}

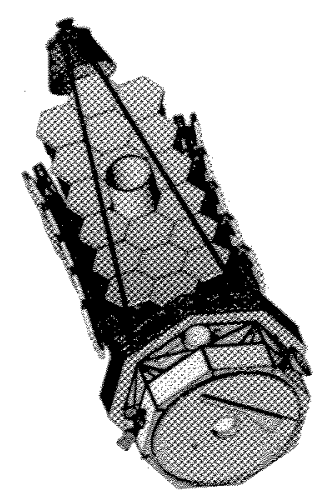

After fairing separation
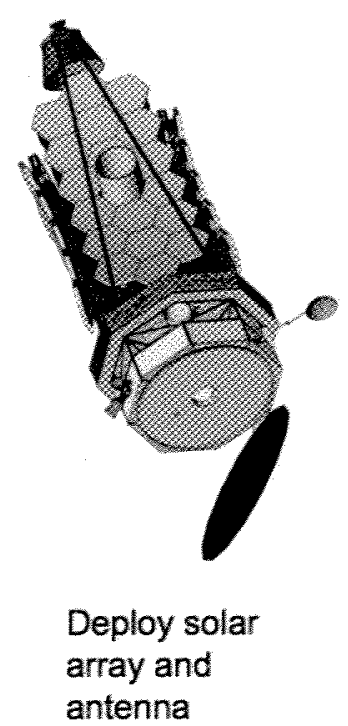
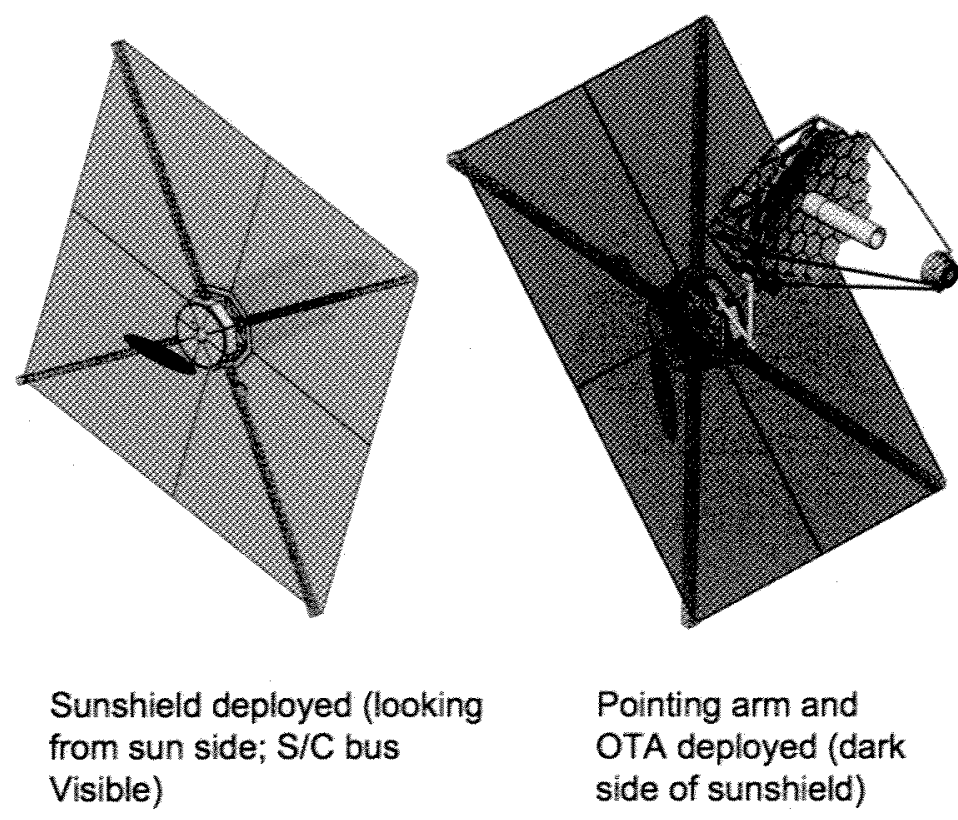
Pointing arm and
OTA deployed (dark
side of sunshield)

Figure 5. Deployment of observatory. Note in the final panel (right) that the OTA sits at the end of a gimbaled pointing arm

\subsection{Sunshield and Stray light}

As previously mentioned, ATLAST-9.2 has significant design heritage from JWST, including the sunshield as shown in Figure 5. A major difference however, is the purpose of the shield. For JWST, the shield blocks light from the sun, while also providing a thermal shield for the cryogenic telescope. However, in the case of ATLAST-9.2, the OTA does not need to be cold, and isothermality at roughly room temperature is more important. This is achieved by the use of distributed heaters. For ATLAST-9.2, the main purpose of the shield is to block light from the sun, earth and moon from 
entering the telescope. Conventional UV/optical telescopes, such as HST, have a familiar cylindrical tube acting as a light baffle. The deployment of a cylindrical structure of size $\sim 10 \times 20 \mathrm{~m}$ around the OTA is somewhat difficult, so we have baselined an architecture employing a roughly planar sunshield, as shown in Figure 5.

The deployed sunshield consists of four layers of opaque Kapton film with a simple flat square shape, $28 \mathrm{~m}$ on a side. The sun-facing layer is a Kapton-E film substrate with a doped-silicon coating that limits its maximum temperature while providing adequate electrical conductivity for charge bleed off. The back-side has a vacuum-deposited aluminum coating. The remaining three layers are standard black-Kapton. The edges of the sunshield will be chamfered (layer 2 slightly smaller than sun-side layer 1 and so forth) to prevent direct sun illumination of the back layers. To ease manufacture, integration and test (I\&T), and stowage procedures, each of the four layers is subdivided into four quadrants that are connected during I\&T so that each deployed layer is continuous. Four 18-meter booms extend in a cruciform configuration to deploy the membranes. Using lessons learned from the deployable JWST sunshield, the ATLAST-9.2 sunshield has been designed to be as simple as possible, with its planar shape being a primary means of keeping it simple. Further work is needed to determine the optimum way to fold and stow the large four-layer Kapton membranes so that four extendable booms can reliably deploy them.

We have imposed a requirement on the observatory that stray light does not exceed $10 \%$ of the naturally occurring zodiacal light from the sky in the field of view. Stray light is minimized by the baffles on the primary and secondary mirrors (see Figure 2). The trade is to size the baffles to maximize the stray light rejection while minimizing blockage of the clear aperture. An analysis of stray light employed reverse ray tracing, as was done for JWST, and was conducted at two wavelengths: $500 \mathrm{~nm}$ and $121.6 \mathrm{~nm}$. We assumed a black kapton application to the sunshield and a deep black coating on the baffles. The primary and secondary mirrors were assumed to have 5 Angstrom surface roughness and $0.5 \%$ particulate contamination. For $\lambda=500 \mathrm{~nm}$, stray light was computed in the $8 \times 8$ arcmin wide field of view camera in the TMA channel and found to be $\sim 8 \%$ of the in-scene zodiacal light. Carefully designed apertures added to the primary and secondary image planes can further reduce the stray light by a factor of $\sim 3$. In the UV, stray light was computed for a $20 \times 20$ arcsec FOV in the Cassegrain channel and was found to be $\sim 50 \%$ of the zodiacal level. However, the zodiacal light level at $121.6 \mathrm{~nm}$ is extremely faint, so this result was considered acceptable.

We found that the primary source of stray light was concentrated near the telescope FOV; ie. nearly on-axis. Only an extremely long $(\sim 100 \mathrm{~m})$ and impractical cylindrical tube would help block such stray light. Furthermore, slightly increasing the diameter of the secondary mirror baffle, resulting in only a few percent loss in collecting area, could provide the equivalent stray light rejection of a long tube around the OTA. Consequently, we have adopted the planar sunshield for ATLAST-9.2.

\subsection{Attitude Control System (ACS)}

The ATLAST-9.2 ACS is a relatively complicated, distributed system. The main components of the overall ACS include: (1) the Fine Guidance Sensor integrated into the science payload, (2) the Disturbance Isolation and Precision Pointing System (DIPPS) between the science payload and the Pointing Arm, (3) Pointing Arm between the DIPPS and the Sunshield/SC Bus, (4) Coarse pointing system on the SC bus that includes gyros, star trackers, Reaction Wheel Assemblies (RWA) and sun sensors, (5) a propulsion system that provides attitude control in certain situations, and (6) Software resident on the SC Bus C\&DH system that runs the ACS algorithms, which enable the ACS components to work together as intended.

The SC bus and Sunshield are always oriented at a 45-degree angle to the sun line, although they are free to rotate any amount about the sun-line as long as this does not result in earth- or moon-light hitting the Science Payload. The Pointing Arm has two roles: one is to pitch the science payload so it can see any target located from 45 to 180 degrees from the Sun, and the other is to permit the telescope to roll \pm 22.5 degrees about its boresight. This feature allows the observatory to observe the same sky location for a long period of time, since the SC bus and sunshield need to rotate about 1 degree per day about the ecliptic pole to follow the apparent motion of the sun.

The DIPPS (Figure 6), which is located between the Pointing Arm and the Science Payload, also has two roles. First, it isolates the science payload from SC bus disturbances, such as vibrations from the Reaction Wheel Assemblies (RWA). Second, it provides fine pointing control for the science payload, which requires a pointing stability of 1.3 milli-arcsec. 


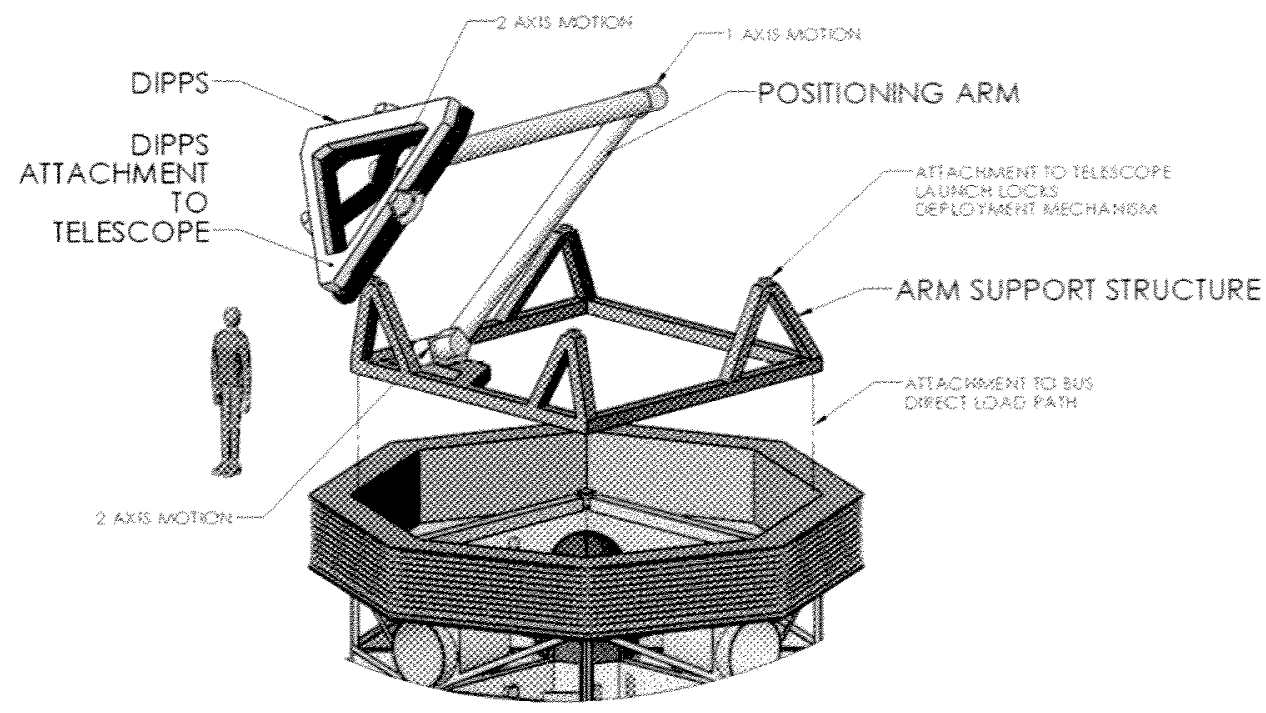

Figure 6. The components of the Disturbance Isolation and Precision Pointing System (DIPPS). The structure just below the arm support structure houses the deployable booms and sunshield (not shown here). The spacecraft bus is right below this.

To start an observation, the star trackers, gyros, and RWA first roll the whole observatory to the proper orientation about the sun line, and then the sensors and motors in the rotary joints of the pointing arm move the telescope to the desired elevation, as well as to the desired roll around the target boresight. Once the pointing has settled, guide stars are acquired in the FGS and tracking begins. Then, the Precision Pointing System of the DIPPS takes over by having the Voice Coil Actuators (VCA) in the DIPPS use the FGS information to initiate a fine track function to minimize the apparent motion of the guide star centroid for the duration of that science exposure. If the linear displacement sensors (which are Differential Impedance Transducers) in the DIPPS indicate that the contactless voice coil actuators (VCA) are gradually approaching their travel limits, then the ACS software commands the RWAs to produce a small, gradual change in the orientation of the SC bus. This causes the DIPPS VCAs to move back near the center of their travel as they adjust to minimize the apparent movement of the guide star centroid. This feedback loop between the FGS, DIPPS, and RWA continues until the end of the science exposure or observations. Disturbance free systems have been studied ${ }^{7}$, but this is certainly a critical technology that needs further development.

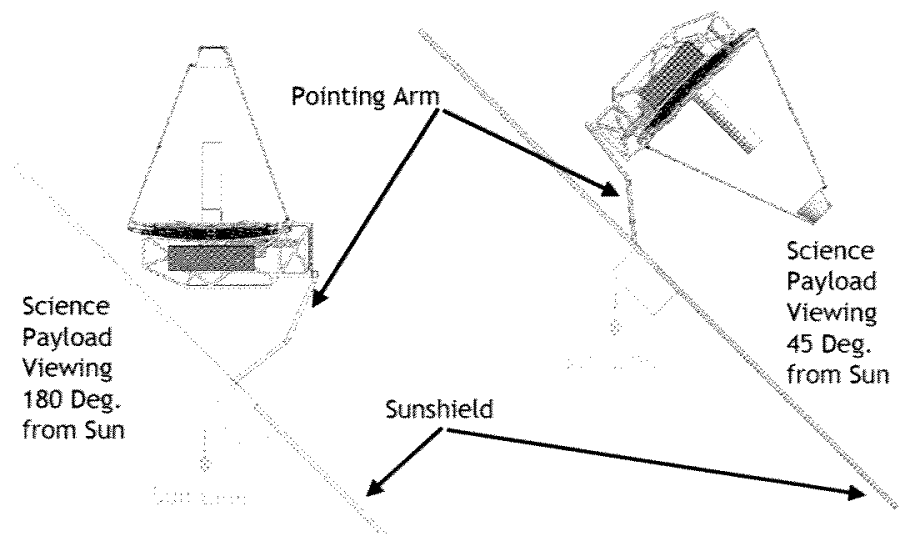

Figure 7. The ATLAST-9.2 Pointing Arm allows telescope pointing 180 degrees from the sun line (left) and also 45 degrees from the sun line (right). Note that the orientation of the sunshield relative to the sun line is fixed at 45 deg. 
At SEL2, occasional small maneuvers are required for station-keeping and unloading of momentum. However, ATLAST-9.2 is able to unload momentum in two axes by using the pointing arm to move the telescope center of mass relative to the center of pressure (from solar photons). Also, ATLAST-9.2 always has the same projected area of sunshade facing the sun. The net result is that fewer momentum-unloading burns are required for ATLAST-9.2 (compared to JWST). Using the ATLAST-9.2 pointing arm and planar sunshade, more than half of the sky is visible at any time, and the orbit allows all of the sky to be seen every 6 months. In Figure 7, we sketch how the center of mass of the observatory can be adjusted by use of the pointing arm, while allowing pointing angles of 45 to 180 degrees from the sun.

\subsection{Spacecraft bus}

The ATLAST-9.2 spacecraft (SC) provides the observatory with power, communications, propulsion, thermal control, and attitude control. As shown in Figure 5 and 7, the SC is always on the sunward side of the sunshield. Halo orbits can be designed at SEL2 that have no eclipses by the earth or moon for 10 years or more, so there is an uninterrupted source of sunlight impinging on the solar array. In addition to the deployable solar array, the SC bus controls the High Gain Antenna (HGA) on a gimbal arm.

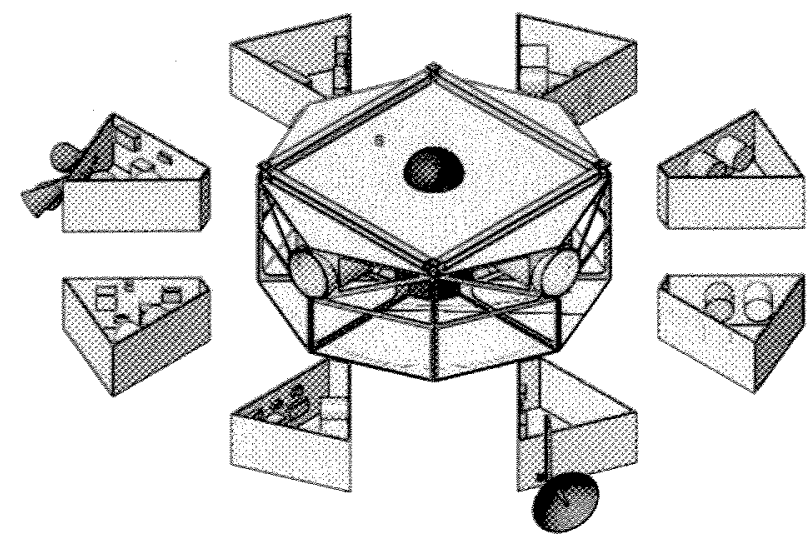

Figure 8. An exploded view of the SC bus, showing the orbital replacement units (ORUs). Note that in this view, the sunshield compartment (with deployable booms) is shown above the SC bus.

The $\mathrm{SC}$ bus is shown in Figure 8. Various spacecraft components are contained in removable Orbital Replacement Units (ORUs), making these components easier to service. Instead of replacing failed boards or power supplies, etc, which often are enclosed deep within the hardware, the notion here is just to remove and replace the whole ORU.

A propulsion system is a required to keep ATLAST-9.2 on its proper trajectory to SEL2, and once there, to provide station-keeping to maintain the observatory in this unstable orbit for the remainder of the mission. The largest single propulsion requirement is to correct for dispersions in the trajectory within 24 hours of separating from the launch vehicle. To reduce propellant mass, this maneuver uses higher performance bipropellant thrusters. However, the delta$\mathrm{V}$ required for station-keeping is better supplied with smaller monopropellant thrusters, which are more controllable and less contaminating. Hence, we have adopted a dual propulsion system of bipropellant and monopropellant thrusters. All thrusters are located on the sunward side of the sunshield in order to prevent contamination of the telescope optics. The propulsion tanks are designed to allow re-fueling.

\section{SERVICING}

We adopted the objective of a 25 -year mission life for the ATLAST-9.2m observatory, and hence designed the facility to be capable of being serviced. Key design considerations included modularity, function during servicing, operable interfaces, and accessibility by robots (or astronauts).

External access to all the instruments is provided from behind the OTA structure (see Figure 2). Each instrument can be individually removed by releasing latches on the instrument structure and sliding the instrument out in a single direction. The latch design self-aligns the replacement instrument as part of installation. The modular design of the spacecraft bus provides access for removal along a radial direction for each module (Figure 8). 
The ATLAST-9.2m design concept includes two docking ports for a maintenance vehicle, one on each side of the sunshield. Each port has rendezvous aids, docking latches, and electrical connections to the maintenance vehicle for power and shared control. The ports are positioned to allow the articulated mechanisms of the robotic maintenance vehicle to perform the required kinematic manipulation of the removable modules. The spacecraft bus propulsion system could have connections, valves, and instrumentation to support refueling by the maintenance vehicle.

\section{SUMMARY}

ATLAST-9.2 is a concept for the next UVOIR flagship observatory and is based on heritage from JWST. This observatory, with diffraction-limited imaging at $\lambda=500 \mathrm{~nm}$ and significant light gathering power, is capable of addressing a wide range of topics at the forefront of modern astrophysics. Most of the required technologies are already in hand, or are considered to be very achievable extensions of the current state of the art. Progress is needed in improving the performance of detectors and optics in the ultraviolet, the surface figure of mirror segments for the primary mirror, the disturbance isolation system, and development of internal coronagraphs and/or an external occulter for high contrast imaging of exoplanets. A technology plan has been put in place ${ }^{8}$ to address these issues, and move forward to consider the next large space observatory to follow HST and JWST.

\section{REFERENCES}

[1] Postman et al, "Science with an 8-meter to 16-meter optical/UV space telescope," Proc. SPIE 7010, 701021 (2008).

[2] Stahl, H. P., "Design of an 8-meter monolithic UV/OIR space telescope", Proc. SPIE 7436, 743609 (2009)

[3] United Launch Alliance website: www.ulalaunch.com

[4] Dahl, R., "Lightweight Glass 1.4m Primary Mirror Segment Assemblies for MMSD", NASA Mirror Technology Days, June 9, 2010.

[5] Dean et al, "Phase Retrieval Algorithm for JWST Flight and Testbed Telescope", Proc. SPIE, 6265, 626511 (2006)

[6] Feinberg et al, "Large Segmented UV-Optical Space Telescope Using a Hybrid Sensor Active Control (HSAC) Architecture", Proc. SPIE 7436, 743608 (2009)

[7] Pedreiro, N., "Spacecraft Architecture for Disturbance-Free Payload", Journal of Guidance, Control and Dynamics, Vol 26, No. 5, 794 (2003)

[8] Hyde, T. and Postman, M., "Technology Development Project Plan for ATLAST: A Roadmap for UVOIR technology 2010-2020", (2009) www.stsci.edu/institute/atlast/index_html_ATLASTMissionConceptStudy_Page 